\title{
Beteiligungskapital für Technologieunternehmen (TU) - Motor für den Aufbau innovativer wettbewerbsfähiger Wirtschaftsstrukturen -
}

\author{
Michael Groß
}

\section{Ausgangssituation und Problem- darstellung}

Der Prozeß des weltweiten Wandels von großen, i. d. R. kapitalintensiven und wenig flexiblen Wirtschaftsstrukturen, zu kleinen und wettbewerbsfähigen TU, ausgestattet mit hochqualifizierten Arbeitskräften, modernsten Basistechnologien und hoher Flexibilität am internationalen Markt, prägt den Übergang der westlichen Industrienationen vom 20. zum 21. Jahrhundert, mit all seinen politischen, wirtschaftlichen und sozialen Folgen. Dabei bilden mehr als 30 Mio. Arbeitslose im EUBereich, davon durchschnittlich $4 \mathrm{Mio}$. in Deutschland, nicht nur sozialen Sprengstoff, sondern zugleich die Basis für neue Denkansätze und Wege.

Der Erfolg bei der Gestaltung der eigenen wirtschaftlichen und sozialen Zukunft wird maßgeblich von den erreichten, international ausgerichteten Markt- und Wettbewerbspositionen sowie von der Effizienz und dem Tempo des Aufbaus einer neuen technologieorientierten Unternehmenslandschaft abhängen. Die Innovation und deren Integration in neue Geschäftsfelder ist dabei ein wichtiger Indikator für die Stärkung der Wettbewerbsfähigkeit und die Jobcreation. Die marktorientierte Nutzung vorhandener sowie die Etablierung neuer Innovationspotentiale, in Form von TU, stehen folglich im Mittelpunkt und sollen den Trend zur Verlagerung kapitalintensiver Industriearbeitsplätze aus Europa, insbesondere Deutschland und anderen hochentwickelten Industrieländern, in sogenannte Billiglohnländer kompensieren und parallel durch die Integration modernster Technologien in die „alten Industriestrukturen“ umkehren.

Insbesondere die Schaffung von neuen Jobs, als Basis fuir weiteren Wohlstand, hat in den EU-Staaten in den 90er Jahren erheblich an Bedeutung gewonnen, da der technologiebedingte Strukturwandel zu erheblichen Arbeitskräftefreisetzungen geführt hat, der in Ostdeutschland sowie wesentlich dramatischer in Osteuropa zusätzlich durch den Transformationsprozeß von der Plan- zur Marktwirtschaft beeinflußt wurde. Während es nach einer Studie von Ernst \& Young in den USA in den letzten 30 Jahren gelang, 60 Mio. neue Jobs, davon seit 1992 fast 14 Mio. Jobs vordergründig in wachstumsorientierten High-tech Unternehmen, zu schaffen, ist dieser Prozeß innerhalb der EU eher als stagnativ bzw. rückläufig zu werten. ${ }^{1}$ Dabei sind ca. 30 Mio. US-Jobs als nachhaltig zu betrachten und trotz der Zweifel am praktizierten US-Ansatz Jobs durch Lohndumping und fehlende soziale Absicherungsmechanismen wettbewerbsfähig zu halten, haben die USA dem 350 Mio. Menschen umfassenden EU-Markt einiges voraus.

Die Schaffung neuer technologieorientierter Unternehmensstrukturen und die Bereitstellung oder Entwicklung der dazu erforderlichen materiellen und finanziellen Potentiale bilden folglich den Kern aller Bemühungen der Europäer zur Sicherung von Wohlstand durch Innovation.

\section{Ausgewählte Spezifika von TU}

Zunächst sei vermerkt, daß sich die weiteren Ausführungen generell auf den Bereich der kleinen und mittleren TU beziehen, die auch als junge TU (nicht älter als 10 Jahre) bezeichnet werden. Gerade dieser Bereich innovativer Unternehmen stellt in wachsendem Maße in Deutschland und der Europäischen Union die neue, international wettbewerbsfähige Unternehmensgeneration dar. Diese TU gliedern sich in die, keinesfalls als homogen zu bezeichnende, Gruppe der KMU ein, die einerseits nach Größenmerkmalen und andererseits nach dem Innovationsgrad charakterisiert werden. In der Praxis gängige Unterscheidungsmerkmale von KMU zur Unternehmensgröße sind beispielsweise:

- Unternehmen mit weniger als 500 Beschäftigten bzw. weniger als 100 Mio. DM Umsatz, entsprechend dem analytischen Ansatz des Institutes für Mittelstandsforschung;

- Unternehmen mit weniger als 250 Beschäftigten und einem Jahresumsatz von weniger als $40 \mathrm{Mio}$. ECU oder einer jährlichen Bilanzsumme von weniger als 27 Mio. ECU, entsprechend der Auffassung der Europäischen Union, insbesondere der zuständigen Direktion 23 furr Unternehmenspolitik;

- Unternehmen mit weniger als 50 Mitarbeitern und einem Jahresumsatz von weniger als $7 \mathrm{Mio}$. ECU sowie einer Bilanzsumme von weniger als $5 \mathrm{Mio}$. ECU, gemäß den Vorgehen des BTU-Programmes (Beteiligungskapital für Technologieunternehmen), das vom Bund getragen wird.

Letzerer Ansatz gilt folglich für die betrachtete Gruppe der TU.

Charakterisiert man die Unternehmensgröße in Abhängigkeit von der phasenorientierten Entwicklung, lassen sich folgende Größenkategorien für TU entwickeln, die 
in der Praxis anzutreffen sind und von dem o. g. Basisansatz abweichen:

- Seed-Phase, i. S. der frühen Gründungs- und Entwicklungsphase

max. 10 Beschäftigte, max. 1 bis 3 Jahre, Umsatz weniger als 1 Mio. ECU p. a.,

- Start-up-Phase, i. S. der ersten Aufbauphase max. 20 Beschäftigte, max. 2 bis 6 Jahre, Umsatz weniger als 5 Mio. ECU p. a.,

- Expansion-Phase, i. S. der ersten Wachstumsphase max. 50 Beschäftigte, max. 3 bis 10 Jahre weniger als 10 Mio. ECU p. a.

Die angegebenen Größenkriterien sind als fließend, nach oben offen, zu bezeichnen und können nach $\mathrm{Ab}$ schluß der 90er Jahre, die in Deutschland und Europa zweifellos als Gründerjahrzehnt für neue TU zu definieren sind, einer ersten wissenschaftlich fundierten Analyse zugeführt werden.

Bereits hier wird deutlich, daß die für Europa und Deutschland geltenden Schablonen für KMU den TU in der Realität nicht gerecht werden.

Bezogen auf den Innovationsgrad ist folgende Differenzierung von KMU anzutreffen:

1. Junge innovative Unternehmen, deren Gründung auf neuen Produkt- oder Verfahrenslösungen sowie Dienstleistungen basiert und stetige Innovationsaktivitäten erfordert.

2. Etablierte innovative Unternehmen, die stetigen Innovationszyklen bedürfen, um wettbewerbsfähig zu bleiben.

3. Geringfuigig oder nicht innovative Unternehmen, die keine F/E-Kapazität vorhalten und deren Wettbewerbsposition unwesentlich von Innovationszyklen abhängt.

Klar ist, daß die betrachteten TU der 1. Gruppe zuzurechnen sind, die jedoch zusätzlich folgende ausgewählte Spezifika aufweisen:

a) Hohe Innovationsaktivität verbunden mit erheblichen F/E- und Markteinfuihrungsrisiken;

b) Hoher Eigenkapitalbedarf zur erfolgreichen Realisierung der Gründungs- und Aufbauphase;

c) Hohe Management- und Personalrisiken;

d) Hohe Umsatz-, Ertrags- und Liquiditätsschwäche in Abhängigkeit von den realen Entwicklungs- und Risikophasen;

e) Hohe Kostenrisiken, aufgrund fehlender Erfahrungswerte;

f) Hohe Abhängigkeit von staatlichen Förderinstrumenten sowie Finanzierungs- und Vermarktungsprogrammen zur Sicherung der Anschubphase;

g) Hohe Ausfallrisiken durch starke, etablierte Wettbewerber mit eigenen Produktangeboten;

h) Hohe Insolvenzrisiken durch risikoaverse Bewertung und Begleitung von Fremdkapitalgebern, aber auch unspezialisierten Beteiligungsfinanziers.

Allein diese Aufzählung, die weiter ergänzt werden könnte verweist auf zahlreiche Risikofaktoren, die die
TU von „normalen KMU“ abheben. Ein Teufelskreis tut sich auf, da die Risiken gerade die TU als Garant für Innovation, steigende Wettbewerbsfähigkeit und Jobcreation betreffen und folglich zu Hemmnissen bei der Etablierung der neuen Unternehmensstruktur führen. Ein grundlegender Wandel ist auf dem Sektor der Mechanismen zur Schaffung neuer TU unerläßlich. Der englische Philosoph, Schriftsteller und Politiker „Francis Bacon (1561-1626) hat in seiner Schrift „Of Innovations“ folgenden Satz geprägt, der heute noch gilt: „He that will not apply new remedies must expect new evils, for time is the greatest innovator."

Es ist offensichtlich auch im Jahre 1998 von Bestand, daß die Anwendung neuer Mittel und Wege im wirtschaftspolitischen Bereich einer Basisinnovation im Vorfeld der Schaffung neuer innovativer Unternehmensstrukturen bedarf, um der international verbreiteten Krankheit des Jobdefizites schrittweise Herr zu werden. Andernfalls werden auch wir vor vollendete, möglicherweise unkalkulierbare Tatsachen gestellt. Dabei ist als allgemein anerkannt zu werten, daß das Venture Capital und auch seine klassische Form des Beteiligungskapitals, eine besondere Funktion ausfuillt, die den Prozeß der Etablierung neuer TU bereits positiv beeinflußt hat und auf Grund seiner Stellung als Leadinvestor wesentlich stärker beeinflussen wird.

\section{Beteiligungskapital und dessen Funktion}

Ausgehend von dem inhaltlichen Ansatz, die Rolle und Funktion des VC beim Aufbau neuer TU zu betrachten, soll weitgehend auf die Darstellung der historischen Entwicklung des VC sowie der einzelnen Gruppen verzichtet werden. Jedoch erscheint es notwendig, einige Ausfuihrungen zu Aspekten der inhaltlichen Differenzierung zwischen VC und klassischem Beteiligungskapital vorzunehmen.

Danach kann grundsätzlich festgestellt werden, daß Beteiligungskapital nicht in jedem Falle VC (i. S. von Risiko- oder Wagniskapital) darstellt. Dieser Sachverhalt ist aus der Risikoorientierung der Beteiligungsgesellschaften abzuleiten, die eine differenzierte Phasenorientierung sowie unterschiedliche Renditeerwartungen nach sich ziehen. Insofern kann bereits hier festgestellt werden, daß öffentlich getragene VC-Gesellschaften mit Förderauftrag bei der Finanzierung von risikobehafteten TU eine besondere Rolle spielen. Diese Fonds erfüllen in der Praxis verstärkt die Forderung nach der Etablierung neuer, dringend erforderlicher TU und Jobs. Insbesondere die Finanzierung von TU in der Seed-und Start-up Phase ist als Domain der öffentlich getragenen VC-Gesellschaften und Förderinstrumente zu bezeichnen, da aufgrund der objektiv bedingten höheren Renditeerwartungen privater VC-Fonds, ein stärkerer Selektionsprozeß eintritt, der die gesellschaftliche Forderung nach zusätzlichen Jobs durch diese VC-Gruppe nur bedingt erfüllbar werden läßt. Diesen Aspekt kann man zweifellos auf den Bereich der klassischen Fremdkapitalgeber ausdehnen, die jedoch objektiv nicht zu den risikoorientierten Frühphasen- 
finanziers gehören. In der Praxis ist zu beobachten, daß die Banken im Verbund mit Frühphasenspezialisten, wie z. B. der Seed Capital Brandenburg GmbH, stetig steigend und risikoabhängig in die neuen Unternehmensstrukturen investieren. Die sogenannte Bankenschelte konnte objektiv nie zutreffen, da Satzung und Aufgaben von privaten und auch öffentlich getragenen Banken dem nicht entsprechen.

Folgende Darstellung soll an dieser Stelle auf den engen Zusammenhang von Risiko- und Fremdkapitalbereitstellung verweisen:

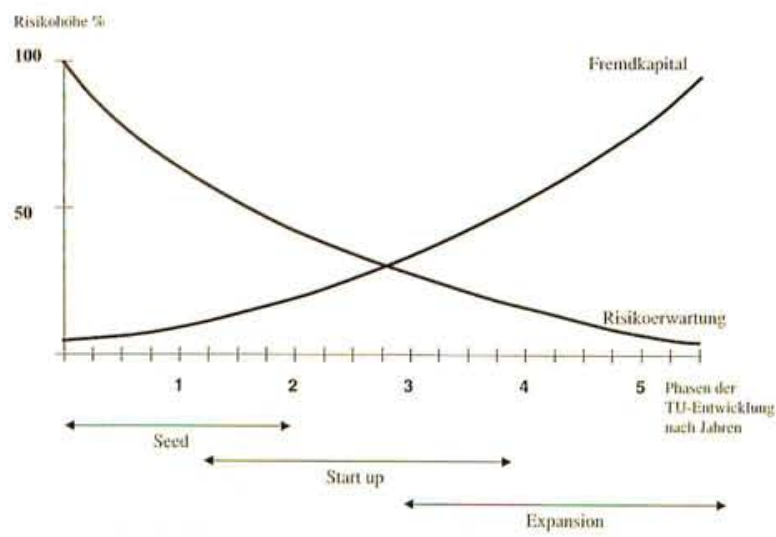

Abb. 1: Fremdkapitalfinanzierung von Technologieunternehmen in Abhängigkeit vom Risiko

Aus dieser Darstellung ist ersichtlich, daß abnehmende Risiken die Fremdkapitalzufuhr erhöhen, d. h. die Frühphase der TU-Etablierung ist durch Eigenkapital und Förderung zu finanzieren. Die Vorzüge des unterschiedlich ausgeprägten Beteiligungskapitals durch die Eigenkapital- und Haftungsfunktion, die Risikoakzeptanzfunktion sowie die Coaching- und Leadinvestorenfunktion haben einen erheblichen Aufschwung der Finanzierung von Unternehmen bewirkt. Darüber hinaus beinhalten VC-Gesellschaften eine Freistellungsfunktion für Sicherheiten zugunsten von Fremdkapitalgebern. Anhand der Darstellung zur Entwicklung des Gesamtportfolios der in Deutschland tätigen Beteiligungsgesellschaften (Abb. 2) soll zunächst die enorme gesamtwirtschaftliche Bedeutung des Beteiligungskapitals nachge- wiesen werden, die sich auch im Ergebnis fallender Eigenkapitalquoten von den 60er Jahren mit durchschnittlich noch rund $30 \%$ auf aktuell fast $18 \%$ vollzogen hat, jedoch unerläßlich für die Abdeckung der Risikophasen von TU ist.

In Summe haben die über 100 im Bundesverband Deutscher Kapitalbeteiligungsgesellschaften (BVK) organisierten Beteiligungsgesellschaften per 31.12 .97 mehr als $7 \mathrm{Mrd}$. DM investiert, per 31.12.98 werden kumuliert mehr als 11 Mrd. DM erwartet. Nach Branchen gegliedert ergibt sich folgendes Bild, das sich aus insgesamt 3496 Beteiligungspartnerschaften ableitet:

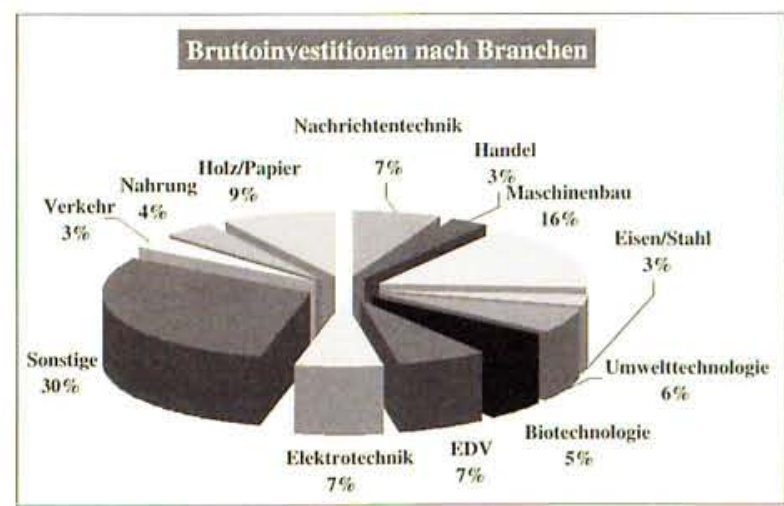

Abb. 3: Bruttoinvestitionen der im BVK organisierten Beteiligungsgesellschaften differenziert nach Branchen (Quelle: BVK-Statistik; Bezugsgröße: BVK-Mitglieder)

Hier wird deutlich, daß auch Ende der 90er Jahre eher traditionelle Branchen überwiegen, was auch nachfolgende Statistik des BVK zu den Investments nach Phasen ausdruickt:

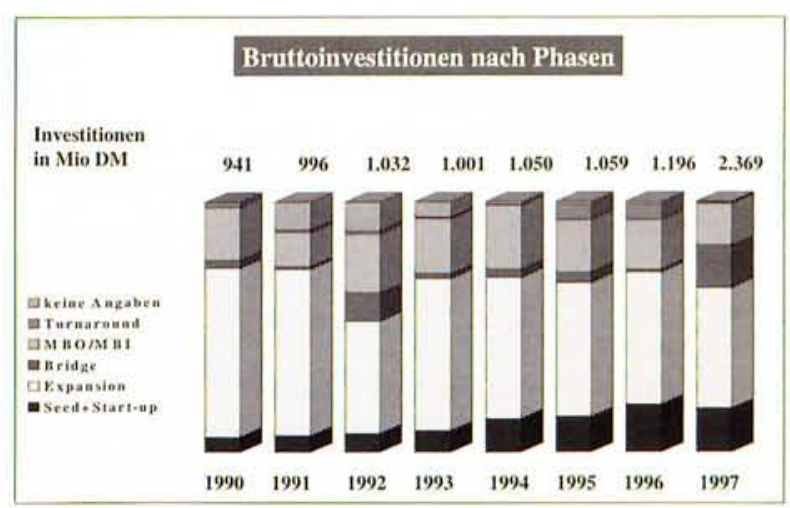

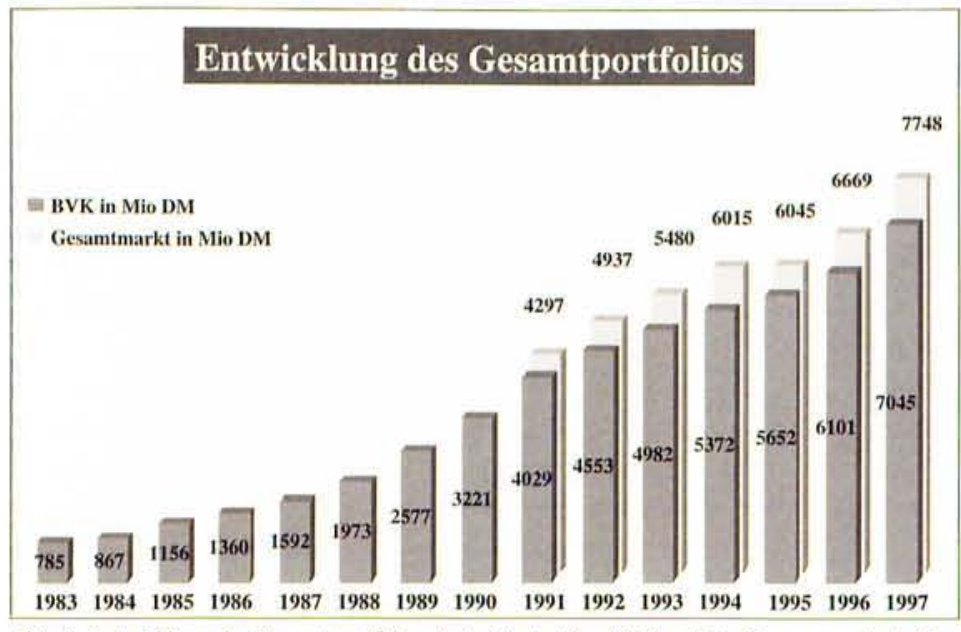

Abb. 2: Entwicklung des Gesamtportfolios der in Deutschland tätigen Beteiligungsgesellschaften (Quelle: BVK-Statistik)
Abb. 4: Bruttoinvestitionen der im BVK organisierten Beteiligungsgesellschaften differenziert nach Phasen der Unternehmensentwicklung (Quelle: BVK-Statistik; Bezugsgröße: BVKMitglieder)

Anhand der o. g. Kenngrößen kann auf die beeindruckende Dynamik auf dem Sektor der Entwicklung des Beteiligungsmarktes mit seiner Kapitalzuführungs- und Koordinierungsfunktion in Deutschland geschlossen werden. 1996, 1997 und auch 1998 sind Rekordzuwächse festzustellen, die einerseits auf den erheblichen Eigenkapitalbedarf im Zuge des industriellen und technologisch bedingten Strukturwandels und andererseits auf die eindeutige Verschiebung der Relation zwischen Fremd- und Eigenkapitalgebern, darunter 
der technologieorientierten VC-Finanziers, hinweisen. Der betrachtete Bereich der TU spiegelt sich prioritär im Anteil der Seed- und Start-up Investments wider, der in Deutschland mit mehr als $12 \%$ eine in Europa ungeschlagene Größe ausmacht. Deutlich wird, daß der größte Teil des „klassischen“ deutschen Beteiligungskapitals auch 1997 zur Expansionsfinanzierung der etablierten Industrie diente und der Anstieg im Frühphasenbereich eindeutiges Ergebnis der hervorragenden Rahmenbedingungen durch das BTU-Programm ist. Eine Rücknahme dieser als „VC-Risikobegrenzungs- bzw. VC-Investaktivierungsprogramm“ zu wertenden Maßnahmen des Bundes, läßt den Rüickfall in deutsche Finanzierungsnormen erahnen, die schlicht aus der rentabilitätsfeindlichen VC-Risikozunahme abzuleiten wäre.

Diesbezüglich soll in Ergänzung zu den o. g. Angaben zum deutschen Kapitalbeteiligungsmarkt auf die Entwicklung in Europa, gemäß den evca-Schätzungen per 31.12.97 und die Veränderungen gegenüber 1996 verwiesen werden.

Kumuliertes Fondsvolumen - 82,6 Mrd. ECU (+ 41 \%) Zufluß neuer Fondsmittel - 20,0 Mrd. ECU (+151\%) Neubeteiligungen $\quad-9,7 \mathrm{Mrd}$. ECU (+ $42 \%)$ Anzahl neuer Beteiligungen- 6252 (davon $4762 \mathrm{KMU}$ ). ${ }^{2}$

Bezogen auf die Finanzierungsphasen belegt der Frühphasenbereich nur $7,4 \%$, entsprechend 711 Mio. ECU des investierten Gesamtvolumens, dagegen machen $\mathrm{MBO} / \mathrm{MBIs}$ mehr als $50 \%$ und fast $4,5 \mathrm{Mrd}$. ECU aus. Interessant ist jedoch, daß die Early-Stage-Investments in 1187 Unternehmen flossen, d. h. jede 4. Beteiligung ging an zukunftsträchtige TU. Trotzdem war noch 1997 kein eindeutiger Trend zu mehr Technologiewerten, analog den US-Entwicklungen, zu erkennen, der jedoch als notwendig anzusehen ist.

Das VC- sowie Beteiligungskapital hat seine Funktion beim Aufbau der neuen industriellen Strukturen zwar gefunden, der notwendige europaweite qualitative Sprung ist trotz allen Wachstums jedoch noch nicht zu verzeichnen.

\section{Beteiligungskapital und Technologie- förderung}

Ausgehend von der Tatsache, daß technologieorientierte Gründungen in Deutschland, Europa und den USA bis zum Ende der Start-up oder Aufbauphase, zwischen 5 und 15 Mio. DM Kapital benötigen, wird neben dem Einsatz von VC generell die Einbindung weiterer Finanzmittelqualitäten in den einzelnen Entwicklungsphasen der TU erforderlich. Daraus resultiert, daß eine gesicherte Gesamtfinanzierung durch das TU-Management auch dem VC-Geber nachzuweisen ist. Die Funktionsausübung des VC hängt folglich im wesentlichem Maße von weiteren Entscheidungsträgern ab, die zur Gewährleistung des Finanzierungsmix für TU, der sich wie folgt verallgemeinern läßt, erforderlich wird:
1. Gründungsfinanzierungsmix von TU (Seed-Phase) zur Darstellung eines Kapitalbedarfes zwischen 1 Mio. DM und 2,5 Mio. $\mathrm{DM}^{3}$

VC-Eigenkapital

Zuschüsse Land/Bund

Fremdkapital

$400-1000$ TDM

Eigenmittel

-700 TDM

200 - 500 TDM

$100-300$ TDM

\section{Folgemix zur Finanzierung der 1. Start-up Phase}

\author{
VC-Leadinvestor \\ VC-Cofinanzier \\ Zuschïsse $^{4}$ \\ Fremdkapital \\ Eigenmittel Gründer \\ Umsätze
}

Die o. g. praktisch durch die Erfahrungen der SCB-GmbH untersetzten Durchschnittswerte zeigen einerseits den tendenziell wachsenden Kapitalbedarf nach Phasen der TU, der prioritär durch VC-Partner, Zuschiusse und generell ansteigend nach Phasen durch Fremdkapital und den Cash-flow der Unternehmen abgedeckt wird. Andererseits werden die notwendigen Entscheidungsfelder, die durch das TU-Management anzusprechen und zu sichern sind, aufgezeigt. D. h., daß die einzelnen Innovations- und Vermarktungsphasen der TU paßfähig für die verschiedenen Finanzmittelgeber vorzubereiten und inhaltlich zu erläutern sind. Die real anzutreffende Vision eines TU-Unternehmers muß parallel in den spezifischen Focus der Entscheidungsträger über deren Finanzierung transformiert werden. Die unter Pkt. 2 aufgezeichneten Risiken von TU beginnen bereits zu wirken, da zeitlich bedingte Wettbewerbsvorteile aufgrund längerer Prüf- und Entscheidungsphasen der different bewertenden Finanzierungspartner verloren gehen. Das Gründungs- bzw. Wachstumsrisiko erhöht sich bereits im Vorfeld der Finanzierung.

Die Technologie- und Wirtschaftsförderung paßt sich gegenwärtig diesem Mißstand schrittweise an, jedoch ist ein qualitativer Sprung in die realen Bedürfnisse der neuen Unternehmensgeneration objektiv noch nicht vorhanden.

Zu viele unabhängig voneinander prüfende und letztlich auch entscheidende Gremien von spezifisch orientierten Fachleuten, machen den Nachweis einer geschlossenen Gesamtfinanzierung für die angestrebten TU zu einer schwer beherrschbaren, z. T. erfolglosen Aktivität. Deshalb sind neue Entscheidungsstrukturen der Finanzierungspartner anzustreben, die effizient und einheitlich agieren. Insbesondere eine Vereinfachung der Entscheidungsstrukturen im Bereich der Fördermittelgeber ist von Relevanz.

Die Ausübung der Rolle der VC-Partner, in ihrer Funktion als Leadinvestor und Eigenkapitalgeber, ist dabei insbesondere in der Seed-Phase von Bedeutung. $\mathrm{Zu}$ schuß- und Fremkapitalgeber orientieren sich in stetig wachsendem Maße am VC-Entscheidungsprozeß, rükken folglich in eine Co-Finanzierungsfunktion. Dies ist zur Sicherung der TU-Etablierung ein objektiv beding- 
ter Weg, der den TU-Anforderungen besser entspricht und die bestehenden Entscheidungsstrukturen in verantwortlicher Weise auf den Prüfungs- und Entscheidungsprozeß der VC-Leadinvestoren aufsetzen läßt.

Der Prüfungs- und Entscheidungsansatz der traditionellen Fremdkapitalgeber ist parallel dringend zu reformieren, um TU-gerechte Entscheidungs- und Finanzierungsprozesse zu gewährleisten.

Grundansatz sollte die Herstellung von zeitlich parallelen und unternehmensorientierten Entscheidungsprozessen sein, die von der gegenwärtigen Form der zeitlich, personell und institutionell differenzierten Entscheidungsfindung über die Finanzierung von TU abweicht. Die Zuordnung der Entscheidungsprozesse sowie der Ebenen der Entscheidung unterliegt, in Abstraktion von weiteren Faktoren, folgenden Differenzierungskriterien:

- Unternehmensart,

- Finanzierungsvolumen,

- Finanzierungszweck (Projekt),

- Mittelqualität.

Dieser Ausgangspunkt gilt zunächst für alle Arten von Kapitalgebern, einschließlich Fördergebern, die daraus die spezifischen Prüfungs- und Entscheidungsmechanismen ableiten.

Der Bereich TU mit seinen besonderen Finanzierungsbediurfnissen und Risiken wird jedoch im Bereich der Feststellung der Unternehmensart sofort im Hochrisikobereich eingeordnet. Die weiteren Entscheidungsprozesse werden bei den notwendigen Eigenkapital- und Fremdkapitalgebern entsprechend betrachtet. VC-Leadinvestoren und TU stehen in Abhängigkeit von zeitlich und inhaltlich kaum beinflußbaren Finanzierungspartnern aus dem Fremdkapital-, aber auch Technologieförderbereich. Letzterer prüft und entscheidet grundsätzlich im Interesse einer zügigen und erfolgreichen Etablierung der TU, jedoch werden projektorientierte, den Finanzierungszweck betreffende Entscheidungen wie z. B.:

Zuschuß- oder Darlehensförderung,

- Liquiditätshilfe,

- Investitionsförderung (GA)

durch personell und zeitlich unabhängige Gremien und Ausschüsse, getroffen. Aus Sicht der VC-Leadinvestoren und TU, sollten i. S. der Sicherung von Zeitabläufen sowie der wirtschaftlichen Stabilität der TU, diese speziellen Entscheidungsfelder durch personell homogene TU-Entscheidungensgremien, die unternehmens- und prozeßorientiert entscheiden, ersetzt werden. Oft scheitern anspruchsvolle Finanzierungsvorhaben von TU und den VC-Leadinvestoren an zu vielen bzw. parallel agierenden Entscheidungsgremien, die den Bereich der Fremkapitalgeber sowie der Förderer betreffen.

Die Reduzierung auf folgende Entscheidungsebenen gegenüber den TU erscheint sinnvoll:
1. Leadinvestor $\quad-\mathrm{VC}$;
2. Fremdkapitalgeber - Banken/Sparkassen;
3. Förderer $\quad-$ Bund/Länder.

Der Leadinvestor gilt als engster Partner des TU, der die mit den TU inhaltlich abgestimmten Konzepte an Cofinanziers und Förderer herausträgt. Der Fremdkapitalgeber mit erstem noch eingeschränkten Engagement, setzt auf dem Konzept auf, trifft seine Entscheidung die in der Regel zeitlich kalkulierbar ist. Die Förderentscheidung wird von einem Gremium von Spezialisten, mit Erfahrungen im TU-Bereich, jedoch unternehmensorientiert fuir alle beantragten Mittelqualitäten getroffen. Die Entscheidung zum Erst- oder Folgeinvestment der TU verkürzt sich nicht nur im Zeitablauf, der Wechsel von der jeweils spezifischen Zweck- und Mittelzuordnungsbetrachtung zur einheitlich zu treffenden unternehmensorientierten Entscheidung, führt zu größerer Effizienz.

\section{Schlußfolgerungen}

Das Bestehen und die Gründung neuer junger Technologieunternehmen hat entscheidenden Einfluß auf die weitere Entwicklung des Technologiestandortes Deutschland und Europa sowie die Erhaltung und Schaffung hochqualifizierter Arbeitsplätze.

Um die positive Entwicklung der 90er Jahre aufrechtzuerhalten und weiter zu forcieren, ist es jedoch erforderlich, die Rahmenbedingungen speziell für die Finanzierung von jungen Technologieunternehmen weiter zu verbessern.

Als wichtige Ansatzpunkte sind dabei zu nennen:

- Erhöhung der Akzeptanz von jungen Technologieunternehmen sowie des damit verbundenen Risikos, insbesondere durch Finanz- und Fördereinrichtungen;

- Anregung und Förderung der Ausgründung aus Hochschulen oder Unternehmen ;

- Unterstuitzung der Gründer von der Erarbeitung der Unternehmenskonzeption bis hin zur Führung und Organisation des Unternehmens (z. B. Coachingfunktion von Beteiligungsgesellschaften, Business Development Center, etc.);

- Stärkere Akzeptanz von VC- oder Beteiligungskapital als „klassisches“ Eigenkapital und ein daraus resultierendes höheres Engagement der Hausbanken, Kreditinstitute und Förderer in risikobehafteten Segmenten;

- Aufrechterhaltung von Programmen zur Refinanzierung von VC-Gesellschaften im besonders risikobehafteten Seed- und Start-up-Bereich von Technologieunternehmen (insbesondere des BTU-Programms);

- Übergang von der projektorientierten Förderpolitik zu einer ganzheitlichen Unternehmensbetrachtung und Ausrichtung der Förderprogramme auf die phasenorientierte Unternehmensentwicklung;

- Bündelung aller für Technologieunternehmen angebotenen Förderprogramme unter dem Dach der auf die Branche sowie die Entwicklungsphasen der TU spezialisierten Prüfungs- und Entscheidungsgremien des Bundes und der Länder; 
- Verkürzung der Entscheidungszeiten der Zuschußgeber, um den technologischen Vorsprung der Unternehmen sowie deren Bestand nicht zu gefährden;

- Stärkere Spezialisierung der Kapitalbeteiligungsgesellschaften auf Entwicklungsphasen und Branchen der TU;

- Schaffung und Akzeptanz der Leadinvestorfunktion zur Koordination der einzelnen Finanzierungsbestandteile;

- Schaffung besserer steuerlicher Rahmenbedingungen für VC- und Beteiligungskapital sowie private Anleger.

Für die weitere erfolgreiche Entwicklung etablierter und neuer Technologieunternehmen ist die Verbesserung bzw. Anpassung der Rahmenbedingungen von entscheidender Bedeutung. Nur durch eine gezielte Spezialisierung aller im Finanzierungssystem junger Technologieunternehmen beteiligter Akteure kann eine optimale Finanzierung und Betreuung der Technologieunternehmen über ihren gesamten Lebenszyklus in der Zukunft gewährleistet werden.

\section{Anmerkungen}

1 Ernst \& Young „European Life Sciences 98“ (1995-1998)

2 Quelle: evca Yearbook 1998

3 Vgl. dazu Deutsche Ausgleichsbank 1998

4 GA-Zuschïsse für investive Maßnahmen ausgenommen

\section{Literatur}

Baier, W.; Pleschak, F: Marketing und Finanzierung junger Technologieunternehmen. Wiesbaden: Gabler, 1996

BVK: BVK - Jahrbuch 1998

Deutsche Ausgleichsbank: Beteiligungsfinanzierung in Technologie-Unternehmen der neuen Bundesländer. Bonn: Deutsche Ausgleichsbank, 1998

evca: EVCA - Yearbook 1998

Kulicke, M.: Die Finanzierung technologieorientierter Unternehmensgründungen. In: Koschatzky, K.: Technologieunternehmen im Innovationsprozeß. Heidelberg: Physica, 1997, S. 127-152

Pleschak, F.; Sabisch, H.; Wupperfeld, U.: Innovationsorientierte kleine Unternehmen. Wiesbaden: Gabler, 1994

\section{Verfasser}

\section{Dr. oec. Michael Groß}

Lehrbeauftragter der Technischen

Fachhochschule Wildau

Geschäftsfuihrer der Seed Capital Brandenburg GmbH

Im Technologiepark 1, 15236 Frankfurt (Oder)

Tel. (03 35) 557-16 90

Fax (03 35) 557-16 99

E-mail: gross@scb-gmbh.de 